\title{
Absorption and Scattering Cross Section of Regular Black Holes
}

\author{
Hai Huang, Ping Liao, Juhua Chen, and Yongjiu Wang \\ College of Physics and Information Science, Hunan Normal University, Changsha, Hunan 410081, China \\ Correspondence should be addressed to Juhua Chen; jhchen@hunnu.edu.cn
}

Received 1 December 2013; Revised 16 January 2014; Accepted 10 February 2014; Published 26 March 2014

Academic Editor: Edward Malec

Copyright (C) 2014 Hai Huang et al. This is an open access article distributed under the Creative Commons Attribution License, which permits unrestricted use, distribution, and reproduction in any medium, provided the original work is properly cited.

\begin{abstract}
By using the partial wave method, we investigate the absorption of massless scalar wave from regular black hole. We numerically carry out the absorption cross section and find that the larger angular momentum quantum number $l$ is, the smaller the corresponding maximum value of partial absorption cross section is. Comparing with Schwarzschild case, the absorption cross section of regular black holes is strengthened in both low and high frequency regions, and the absorption cross section oscillates around the geometric optical value in the high frequency region. Generally speaking, the scattering flux is strengthened and its scattering width becomes narrower in the forward direction. There are obvious contrast of scattering properties of different type of regular black hole.
\end{abstract}

\section{Introduction}

Over the last four decades, the physics of particle scattering from different kinds of black holes was one of the most active topics of strong gravitational fields. Apart from the quasinormal modes which, in principle, can be identified as the poles of the corresponding black hole scattering matrix, the behavior of the cross section with respect to the scattering angle is one of the most interesting features in this area. The key issues around which the physics of black hole scattering centers are related to phenomena such as glory, orbiting, rainbow and super-radiant scattering. Scattering by black holes is of fundamental interest in both black hole physics and scattering theory. The presence of black holes can be inferred only through indirect methods. One of the most useful and efficient ways to study the properties of black holes is by scattering matter waves off them. The study of absorption of waves and particles by black holes and by analogous higher-dimensional objects has received considerable attention, because this topic is directly relevant to numerous fundamental aspects of classical and quantum black hole physics which could permit us to progress in our understanding of spacetime properties (see, e.g., [1-12] and the references therein). By using a numerical method, Okawa et al. [13] show that in a scattering of two black holes in the 5-dimensional spacetime, a visible domain, whose curvature radius is much shorter than the Planck length, can be formed.
Décanini et al. [14] show that the fluctuations of the highenergy absorption cross section are totally and very simply described from the properties of the waves trapped near the photon sphere. Batic et al. [15] show that the case of orbiting scattering of massless spin 0,1 , and 2 particles from Schwarzschild black holes can be characterized by a sudden rise in $\left|R^{l}\right|$ at a critical angular momentum.

In 1968, Bardeen [16] constructed the first example of a regular black hole. A regular, nonsingular geometry with an event horizon, satisfying the weak energy conditions, is necessary to introduce some external form of matter or a modification to gravity. The solution was obtained by introducing an energy momentum tensor, regular and bounded, decaying at infinity, and satisfying the weak energy conditions. It can be interpreted as the gravitational field of some sort of nonlinear magnetic monopole [17]. Bardeens work has been followed by several other examples, motivating deeper analyses of how singularity avoidance may be possible in general [1723]. For a large class of black hole solutions, absence of singularities was related to a change in the topology beyond the event horizon. Borde demonstrated the impossibility of proving general singularity theorems when the strong energy condition or the existence of global hyperbolicity was not assumed [24].

We mainly focus on the scalar scattering process of regular black holes and how black hole interacting with 
the nonlinear electrodynamics affect scalar absorption and scattering. The plan of this paper is as follows. In Section 2, we set up scalar field equation of regular black holes and analyse effective potential. In Sections 3 and 4, we concentrate on the absorption and scattering cross section of the scalar wave from regular black holes. We give a brief conclusion in the last section.

\section{The Partial Wave Method}

2.1. Massless Scalar Field Equation. In different context of gravity coupled to some forms of matter, regular solutions were obtained from a prototypical action of the form [16]

$$
S=\frac{1}{16 \pi} \int d^{4} x \sqrt{-g}(R-\ell)
$$

where $g$ is the determinant of the metric $g_{\mu \nu}, R$ is the scalar curvature, and $\ell$ represents the Lagrangian of the matter fields. For the case of nonlinear electrodynamics, $\ell=\ell(F)$ is a nonlinear function of the electromagnetic field strength with $F=F_{\mu \nu} F^{\mu \nu} / 4$.

The spherically symmetric regular black hole solutions take the form

$$
d s^{2}=f(r) d t^{2}-f^{-1}(r) d r^{2}-r^{2} d \Omega^{2},
$$

where the lapse function $f(r)$ depends on the specific form of underlying matter. In the Bardeen spacetime, the function takes a particularly simple form as [16]

$$
f(r)=1-\frac{2 m r^{2}}{\left(r^{2}+\alpha^{2}\right)^{3 / 2}},
$$

with $\alpha=$ const. and $m$ being the mass of the solution. This implies a specific matter energy-momentum tensor, that is, de Sitter, at the core and vanishes away at infinity as a magnetically charged solution in the context of a specific nonlinear electrodynamics [17] with Lagrangian given by $\ell=$ $\left(3 /\left(2 s \alpha^{2}\right)\right)\left(\sqrt{2 \alpha^{2} F} /\left(1+\sqrt{2 \alpha^{2} F}\right)\right)^{5 / 2}$, where $s=|\alpha| / 2 m$ and $\alpha$ is the magnetic charge. By substituting the metric (2) into Klein-Gordon equation $\square \Psi=0$ and the separation ansatz $\Psi=\left(\psi_{\omega l} / r\right) Y_{l m}(\theta, \varphi) e^{-i \omega t}$, we can obtain the radial perturbation equation for the massless scalar field

$$
f(r) \frac{d}{d r}\left(f(r) \frac{d \psi_{\omega l}}{d r}\right)+\left[\omega^{2}-V_{\mathrm{eff}}(r)\right] \psi_{\omega l}(r)=0,
$$

with the effective scattering potential

$$
V_{\mathrm{eff}}(r)=f(r)\left(\frac{1}{r} \frac{d f(r)}{d r}+\frac{l(l+1)}{r^{2}}\right) .
$$

2.2. Effective Scattering Potential. In terms of the Wheelertype coordinate $x=x(r)$ defined by $d / d x \equiv f(r) d / d r,(5)$ can be rewritten as

$$
\frac{d^{2} \psi_{\omega l}(x)}{d x^{2}}+\left[\omega^{2}-V_{\mathrm{eff}}(x)\right] \psi_{\omega l}(x)=0 .
$$

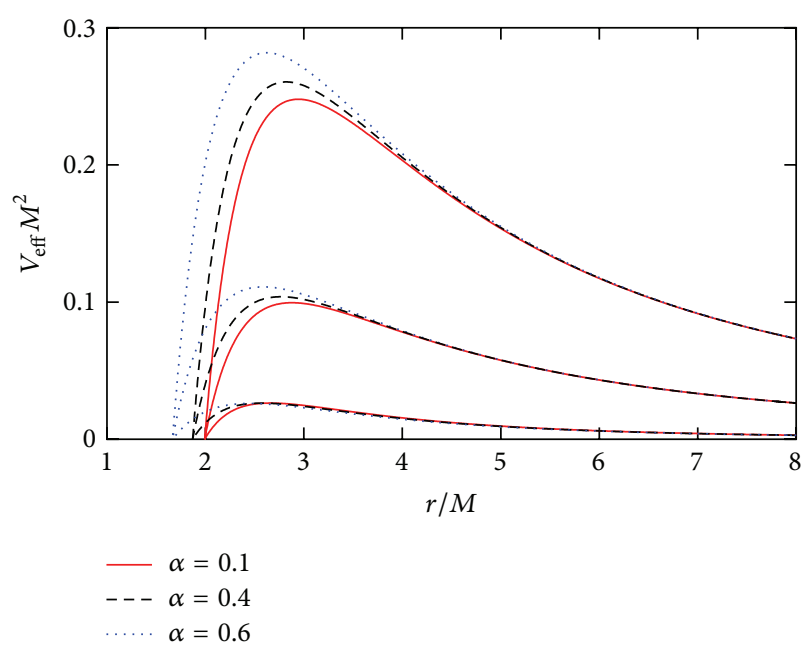

FIGURE 1: The scattering potential $V_{\text {eff }}$ is plotted for different values of the angular momentum $l$ by fixed parameters $M=1, \alpha=0.1,0.4$, 0.6 in the Wheeler-type coordinates. The larger the value of $l$ is, the higher the scattering barrier is.

In Figure 1 we plot $V_{\text {eff }}$ as a function of the Wheeler-type coordinate $x$. It is obvious that the larger the value of $l$ is, the higher the scattering barrier is.

The asymptotic forms of the modes incoming from the past infinity can be written as

$$
\psi_{\omega l} \approx \begin{cases}A_{\omega l}^{\text {tr }} e^{-i \omega x} & \text { for } x \longrightarrow-\infty, \\ A_{\omega l}^{\mathrm{in}} e^{-i \omega x}+A_{\omega l}^{\mathrm{out}} e^{i \omega x} & \text { for } x \longrightarrow+\infty,\end{cases}
$$

with the conserved relation $\left|A_{\omega l}^{t r}\right|^{2}+\left|A_{\omega l}^{\text {out }}\right|^{2}=\left|A_{\omega l}^{\text {in }}\right|^{2}$. The phase shift $\delta_{l}$ is defined by

$$
e^{2 i \delta_{l}}=(-1)^{l+1} \frac{A_{\omega l}^{\text {out }}}{A_{\omega l}^{\text {in }}} .
$$

\section{Absorption Cross Sections}

Based on the quantum mechanics, the total absorption cross section can be written as

$$
\sigma_{\mathrm{abs}}=\frac{\pi}{\omega^{2}} \sum_{l=0}^{\infty}(2 l+1)\left(1-\left|e^{2 i \delta_{l}}\right|^{2}\right),
$$

and we can obtain

$$
\sigma_{\mathrm{abs}}=\sum_{l=0}^{\infty} \sigma_{\mathrm{abs}}^{(l)}=\frac{\pi}{\omega^{2}} \sum_{l=0}^{\infty}(2 l+1)\left|T_{\omega l}\right|^{2},
$$

where $\left|T_{\omega l}\right|$ is the transmission coefficient. Moreover, by considering the Wronskian of two linearly independent solutions of (4) at $x= \pm \infty$, we obtain

$$
1+\left|A_{\omega l}^{\text {out }}\right|^{2}=\left|A_{\omega l}^{\text {in }}\right|^{2} .
$$

Introducing the transmission and reflection amplitudes $T_{l}$ and $R_{l}$ defined by

$$
T_{l}=\frac{1}{A_{\omega l}^{\text {in }}}, \quad R_{l}=\frac{A_{\omega l}^{\text {out }}}{A_{\omega l}^{\text {in }}},
$$




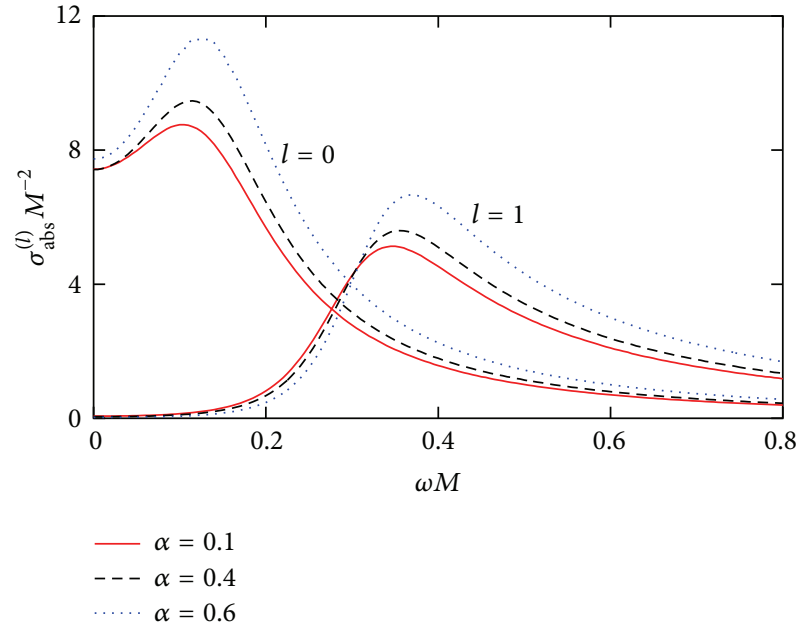

FIGURE 2: The partial absorption cross section is plotted as a function of the parameter $\omega M$ for $l=0,1$ with $\alpha=0.1,0.4,0.6$. The larger $\alpha$ is, the larger the partial absorption cross section is.

we can rewrite (11) in a familiar form

$$
\left|T_{l}\right|^{2}+\left|R_{l}\right|^{2}=1
$$

In order to obtain absorption cross sections of scalar field wave from black hole spacetime, we will solve the radial equation (6) under the boundary conditions (7) and compute the coefficients $A_{\omega l}^{\text {in }}$ and $A_{\omega l}^{\text {out }}$ by matching (8) to get the numerical phase shift. The partial and total absorption cross sections of scalar wave from regular black hole can be simulated immediately. In this study, we adopt the WKB approximation and Pöschl-Teller potential approximation

$$
V_{\mathrm{PT}}\left(x^{*}\right)=\frac{V_{0}}{\cosh ^{2}\left[K\left(x^{*}-x_{0}^{*}\right)\right]},
$$

where $x^{*}$ is the position of the potential peak, and $V_{0}=$ $V_{\mathrm{PT}}\left(x_{0}^{*}\right)>0$ and $-2 K^{2}=V_{\mathrm{PT}}^{\prime \prime}\left(x_{0}^{8}\right) / V_{0}<0$ are the height and the curvature of the potential, respectively $[25,26]$.

The partial absorption cross sections for $l=0,1$ with magnetic charge $\alpha=0.1,0.4,0.6$ are plotted in Figure 2, respectively. It is obvious that the partial absorption cross section tends to zero as $\omega M$ increases. Comparing the curves with a different value of magnetic charge $\alpha$, we can see that the larger the magnetic charge $\alpha$ is, the larger the partial absorption cross section is. It also shows that the difference of the curves is numerically small with respect to their amplitude with larger values of $\omega M$.

In Figure 3 we plot our results for the partial absorption cross section $\sigma_{\text {abs }}^{(l)}$ for $l=0$ up to $l=6$. We see that the partial wave $(l=0)$ contribution is responsible for the nonvanishing cross section in the zero-energy limit. Moreover, for each value of $l>0$, the corresponding partial absorption cross section starts from zero, reaches a maximum value $\sigma_{\text {abs }}^{(l) \max }$, and decreases asymptotically. The larger the value of $l$ is, (i) the smaller the corresponding value of $\sigma_{\mathrm{abs}}^{(l) \max }$ is and (ii) the larger the value of $\omega M$ associated with $\sigma_{\mathrm{abs}}^{(l) \max }$ is. This is all compatible with the fact that the scattering potential $V_{\text {eff }}$ is larger for larger values of $l$.

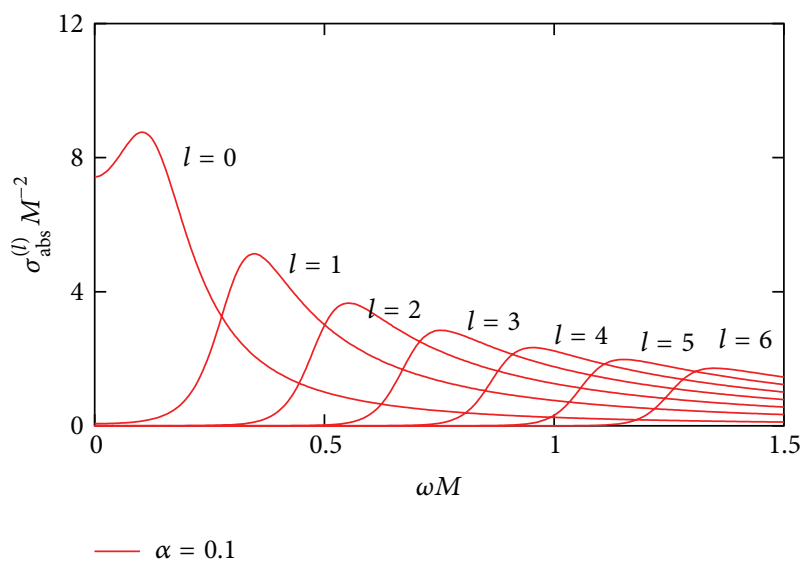

FIGURE 3: The partial absorption cross section is plotted as a function of the parameter $\omega M$ by fixed parameters $M=1$, for $l=0$ to $l=6$ with $\alpha=0.1$. The larger the value of $l$ is, the smaller the corresponding value of $\sigma_{\mathrm{abs}}^{(l) \max }$ is.

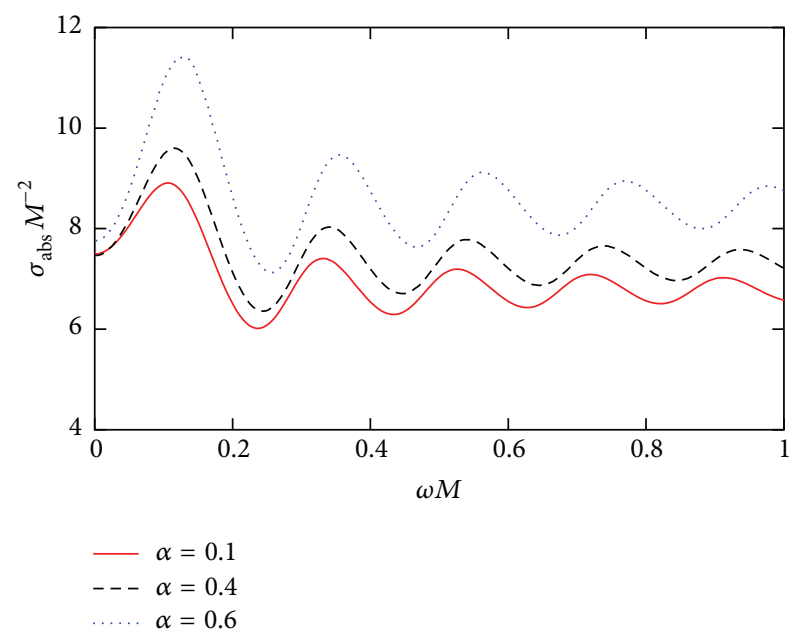

FIGURE 4: Total absorption cross section for a massless scalar field propagating in regular black hole for three values of $\alpha=0.1,0.4,0.6$, respectively. The summation in (10) is performed up to $l=6$. The larger value of $\alpha$ corresponds with the larger value of $\sigma_{\mathrm{abs}}$.

In Figure 4 we plot our numerical results for the total absorption cross section $\sigma_{\text {abs }}$ for $l=0$ up to $l=6$ with $\alpha=0.1$, $0.4,0.6$. We see that the larger the value of magnetic charge $\alpha$ is, (i) the larger the corresponding value of $\sigma_{\mathrm{abs}}$ is, (ii) the smaller the value of $\omega M$ associated with extremum is, and (iii) the larger the corresponding value the total absorption falls approaching is. We can also see that the total absorption cross section $\sigma_{\text {abs }}$ oscillates around the limit of geometrical optics. At the region $\omega M \sim 0.2$, the total absorption sections create a regular oscillation pattern, and when $\omega M \gg 1$, the absorption cross section tends to the geometric-optical limit of $\sigma_{\text {abs }}^{h f}=\pi b_{c}^{2}$, where $b_{c}=r_{c} / \sqrt{f\left(r_{c}\right)}$, and the assumptions on $r_{c}$ imply the existence of a photon sphere located at $r_{c}$ which is the radius of unstable circular null geodesics [27]. 


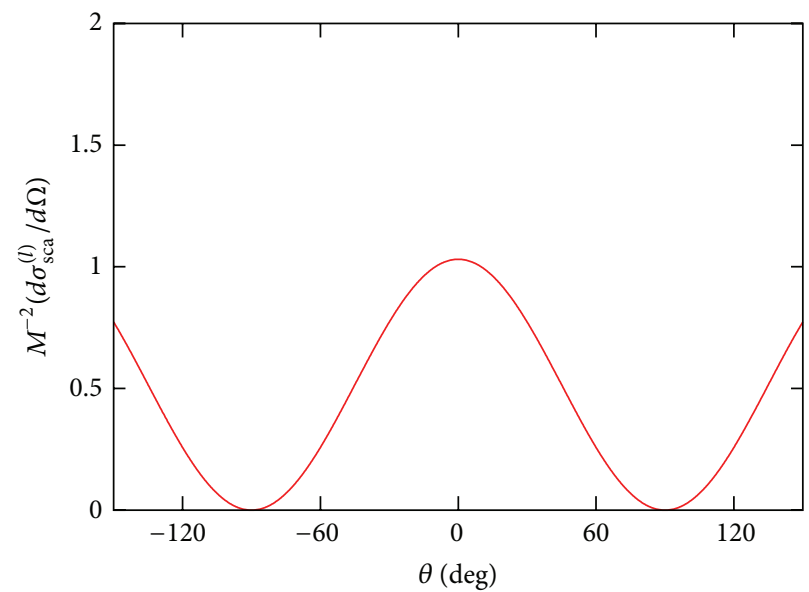

(a) $l=1$

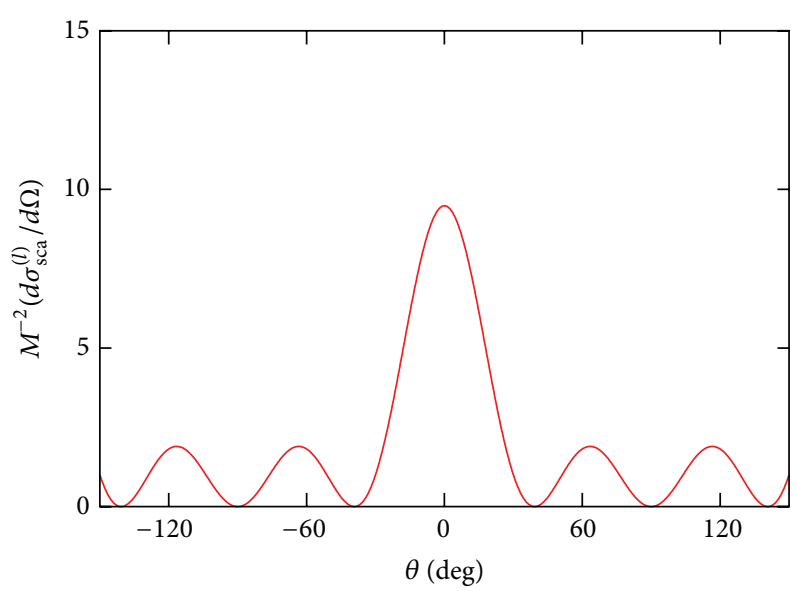

(c) $l=3$

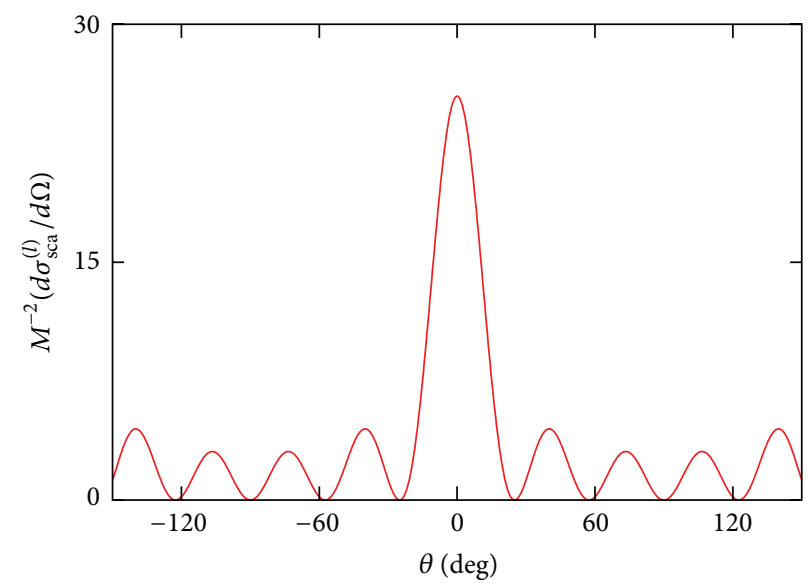

(e) $l=5$

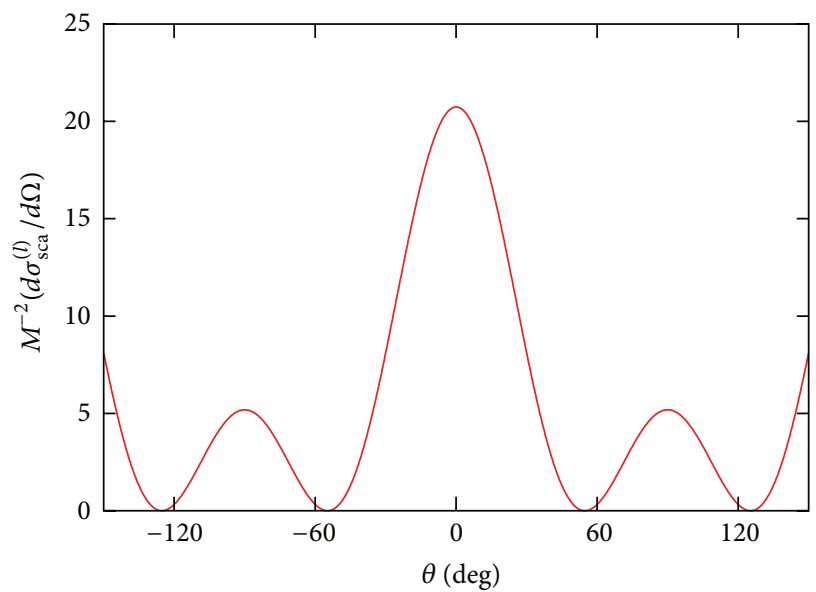

(b) $l=2$

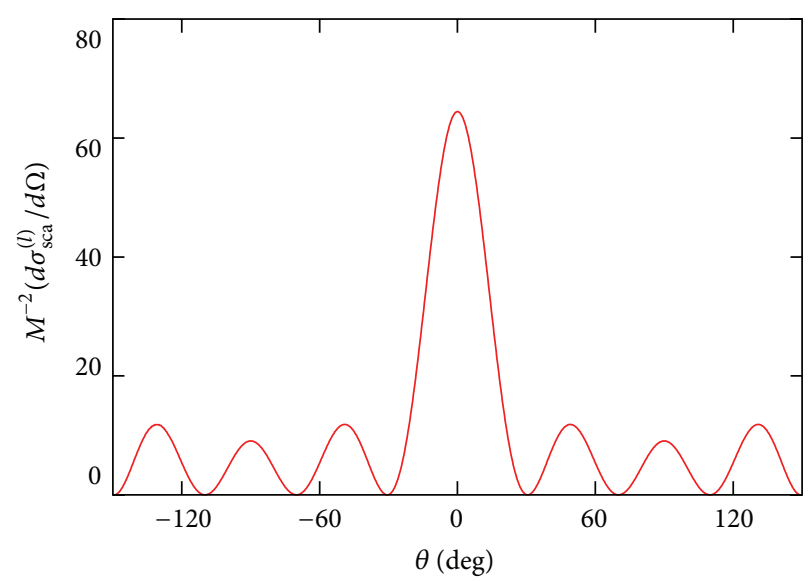

(d) $l=4$

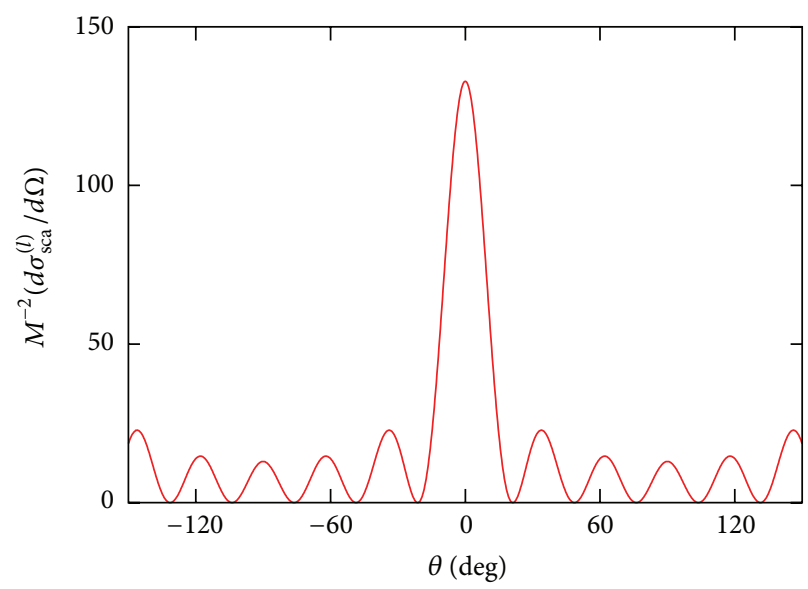

(f) $l=6$

FIGURE 5: The behavior of the partial scattering cross sections $\sigma_{\text {sca }}$, from $l=1$ to $l=6$, at $M \omega=1$ for the scalar wave is scattered from the regular black hole with $\alpha=0.4$. The width of the main scattering angle becomes narrower and the differential scattering cross section becomes higher as partial wave number $l$ increases. 


\section{Scattering Cross Section}

Based on the quantum mechanics, it is well known that the scattering amplitude is expressed as

$$
f(\theta)=\frac{1}{2 i \omega} \sum_{l=0}^{\infty}(2 l+1)\left[e^{2 i \delta_{l}}-1\right] P_{l}(\cos \theta) .
$$

From this scattering amplitude, we can give the differential scattering cross section immediately as

$$
\frac{d \sigma}{d \Omega}=|f(\theta)|^{2} .
$$

At last we can define the scattering and absorption cross section $[28,29]$

$$
\sigma_{\mathrm{sca}}(\omega)=\int \frac{d \sigma}{d \Omega} d \Omega=\frac{\pi}{\omega^{2}} \sum_{l=0}^{\infty}(2 l+1)\left|e^{2 i \delta_{l}}-1\right|^{2} .
$$

In order to simulate the scattering cross sections (17), we must numerically solve differential equation (6) under boundary conditions (7) to obtain numerical values for the phase shifts via (8).

Figure 5 shows the partial scattering cross section, a function of angle for six different partial waves from $l=1$ to $l=6$. By comparing these figures, we can see that (i) the partial flux is scattered preferentially in the forward direction, and a more complicated oscillatory pattern arises as partial wave number $l$ increases. (ii) The width of the main scattering angle becomes narrower and the differential scattering cross section becomes higher as partial wave number $l$ increases. These similar properties are described in $[6,8,10]$ and corresponding physics explanation can be found in [30]. (iii) The oscillation of differential scattering cross section is symmetrical by $\theta=0$ and it has the maximum value along this angle.

The relationship between the logarithm of total scattering cross section and scattering angle $\theta$ can be seen in Figure 6 . We compare the scattering cross sections of the Scharzschild black hole with the regular black hole with $\alpha=0.4$ and 0.6. In forward direction, we find that the scattering flux becomes stronger and the width of scattering cross sections becomes narrower. In other words, the scalar field scattering becomes more concentrated due to the regular black hole. The glory phenomenon exists along the backward direction $[6,8,29,31]$. At fixed frequency, the glory peak is higher and the glory width becomes narrower. So we can find that the glory phenomenon along both the forward and backward directions becomes better for astronomy observation.

Amongst the various models, the lapse function $f(r)$ in (2) has different forms, and some of the solutions considered in this paper are summarized in Table 1.

Table 1, the models of $[16,18-22]$ were constructed in such a way to have Reissner-Nordström asymptotics. The solution $[16,20]$ can be put in a nonlinear electrodynamics framework. The solution given in [20] is a minimal regular solution with the parameter $m$ measuring the mass and the parameter $\alpha$ measuring the deviation of the nonsingular solution from Schwarzschild, which is reproduced for $\alpha=0$. The models

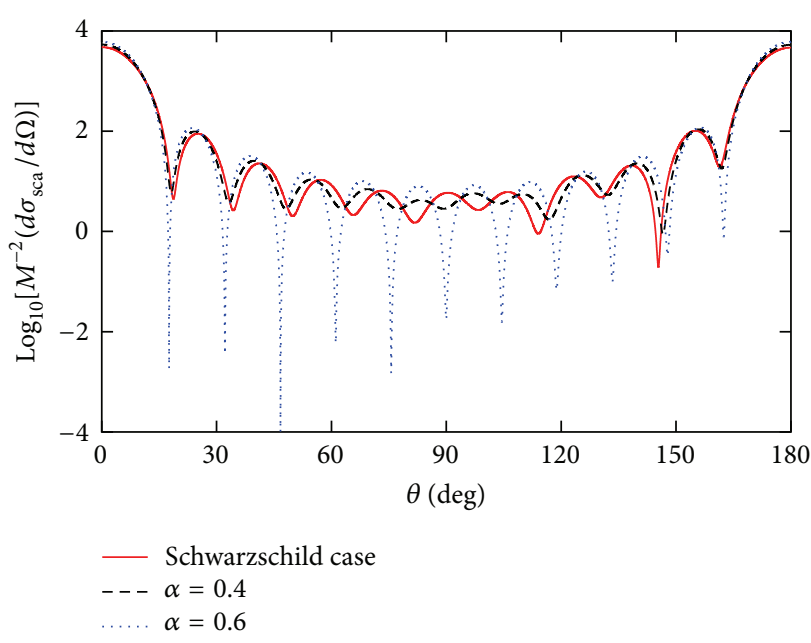

FIGURE 6: The behavior of the total scattering cross sections $\sigma_{\text {sca }}$ at $\omega M=1$ in the range of $0^{\circ}-180^{\circ}$ for the scalar wave is scattered from the regular black hole with $\alpha=0.4,0.6$ ( $\alpha=0$, Schwarzschild case). In forward direction, the scattering flux becomes stronger and the

\begin{tabular}{|c|c|}
\hline Lapse function & Reference \\
\hline (1) $f(r)=1-\frac{2 m r^{2}}{\left(r^{2}+\alpha^{2}\right)^{3 / 2}}$ & {$[16]$} \\
\hline (2) $f(r)=1-\frac{2 m r^{2}}{r^{3}+2 \alpha^{2}}$ & [20] \\
\hline (3) $f(r)=1-\frac{2 m}{r}\left(1-\tanh \frac{r_{0}}{r}\right)$ & [22] \\
\hline (4) $f(r)=1-\frac{4 m}{\pi r}\left(\tan ^{-1} \frac{r}{r_{0}}-\frac{r r_{0}}{r^{2}+r_{0}^{2}}\right)$ & {$[18]$} \\
\hline (5) $f(r)=1-\frac{2 m r^{2}}{\left(r^{2}+q^{2}\right)^{3 / 2}}+\frac{q^{2} r^{2}}{\left(r^{2}+q^{2}\right)^{2}}$ & [19] \\
\hline (6) $f(r)=1+\frac{c r^{2}}{b^{2}}+\frac{\rho_{0} r^{2}}{b^{3}}\left(\frac{b \sqrt{r^{2}-b^{2}}}{r^{2}}+\tan ^{-1} \frac{\sqrt{r^{2}-b^{2}}}{b}\right)$ & [21] \\
\hline
\end{tabular}
width of scattering cross sections becomes narrower.

TABLE 1: Entropy integral between different oarts of radius.

of $[18,19,22]$ were constructed in the context of nonlinear electrodynamics coupled to gravity using different functional forms for $\ell$. The parameter $r_{0}$ in $[18,22]$ is a length scale related to the electric charge and $q$ in [19] is the electric charge itself. The solution proposed in [21] was constructed coupling gravity to a phantom scalar field with a potential $U\left(b, c, \rho_{0}\right)$ where the parameters $b, c, \rho_{0}$ characterize the features of the potential. All the details can be found in the original references.

In Figure 7 we show the absorption cross section for a range of parameters $\alpha=0.1,0.4,0.6$, length scale $r_{0}=0.1,0.2$, 0.3 , and electric charge $q=0.1,0.4,0.6\left(\alpha=0, r_{0}=0\right.$, $q=0$ Schwarzschild case). At fixed $\omega M$, we can see that larger value of parameter $\alpha, r_{0}, q$ corresponding to larger total absorption cross section; the change of total absorption cross section corresponding to $r_{0}$ is relative medial while as $q$ increases, the total absorption cross section increases 


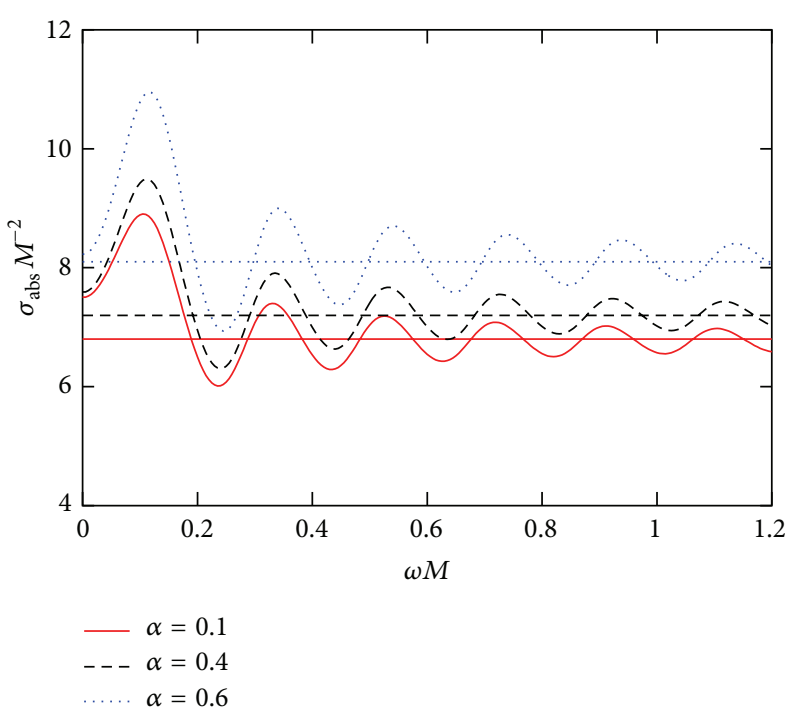

(a) Type 2

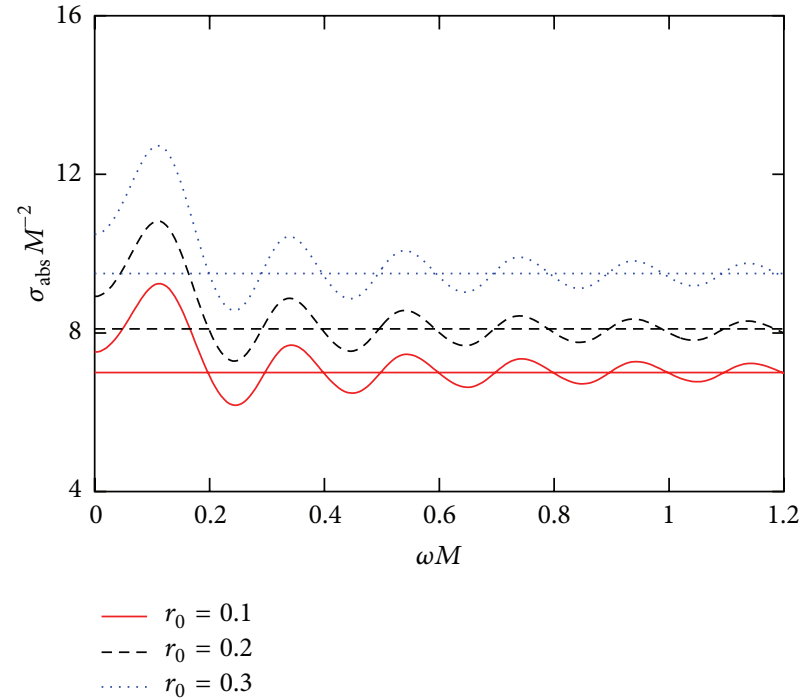

(b) Type 3

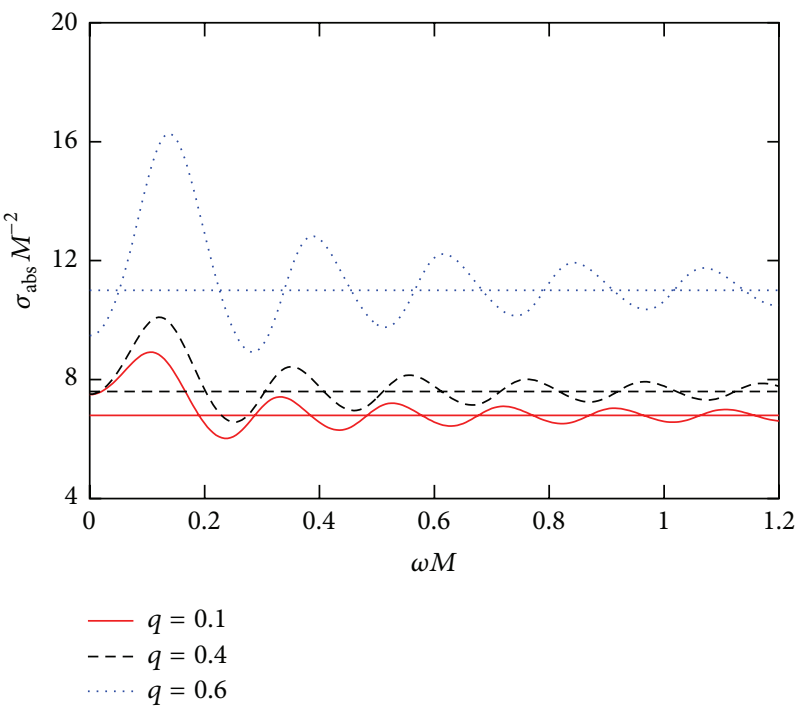

(c) Type 5

FiguRE 7: Total absorption cross section for a massless scalar field propagating in different type of regular black holes for three values of $\alpha, r_{0}$, $q$, respectively. The summation in (10) is performed up to $l=6$. As the frequency increases, the absorption cross section oscillates regularly around the geometric optical value (represented by horizontal lines).

more obviously. As the frequency increases, the absorption cross section oscillates regularly around the geometric optical value.

The scattering cross section of types $2-5$ is single parameter. In Figure 8 we show the scattering cross section for a range of parameters $\alpha=0.1,0.4,0.6$, length scale $r_{0}=0.1,0.2$, 0.3 , and electric charge $q=0.1,0.4,0.6\left(\alpha=0, r_{0}=0, q=0\right.$ Schwarzschild case). Compared to Schwarzschild case, we find these characteristics: type (2) scattering flux is strengthened and its width becomes narrower in the forward direction. The scalar field scattering becomes more concentrated due to the regular black hole. In the backward direction, scattering flux strength becomes greatly different, and when $\alpha=0.4$ scattering flux becomes weaker and narrower, and while $\alpha=0.6$ scattering flux becomes stronger and narrower. Type (3) scattering flux becomes stronger and its width becomes narrower in the forward direction. In the backward direction, scattering flux becomes weaker and narrow. In the angle of $90^{\circ}-180^{\circ}$, the situation is more complicated, and there are some irregular fluctuations; when $r_{0}=0.4$, there is a glory phenomenon. In type (4), when $r_{0}=1.0$, the cross section of scattering flux is tended to be consistent in every direction, and only in the backward direction its strength becomes greater. When $r_{0}=2.0$, in the forward direction, the scattering flux becomes stronger and its width becomes narrower, while in the backward direction it becomes weaker and narrower. In type (5), when $r_{0}=0.4$, scattering flux is similar to type (4), strengthened in the forward direction and 


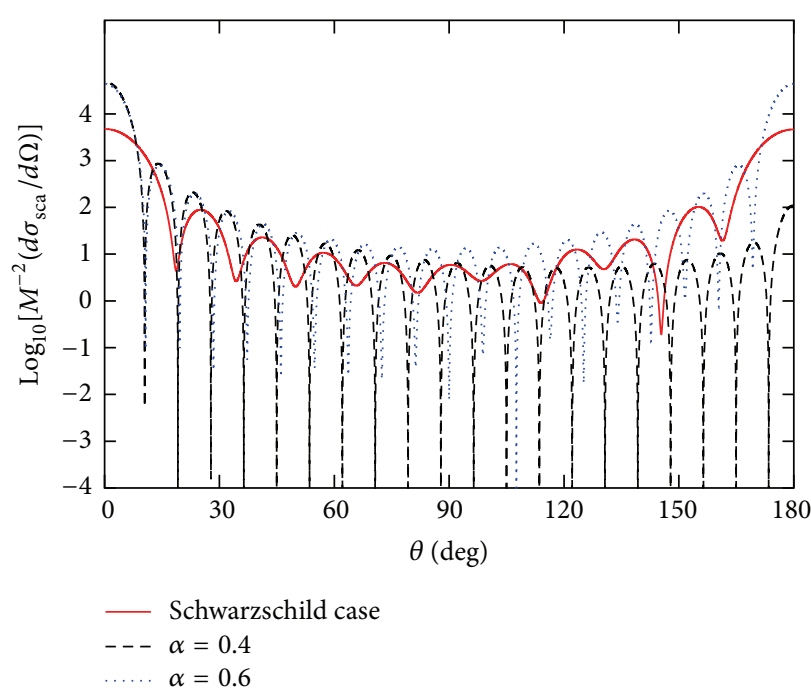

(a) Type 2

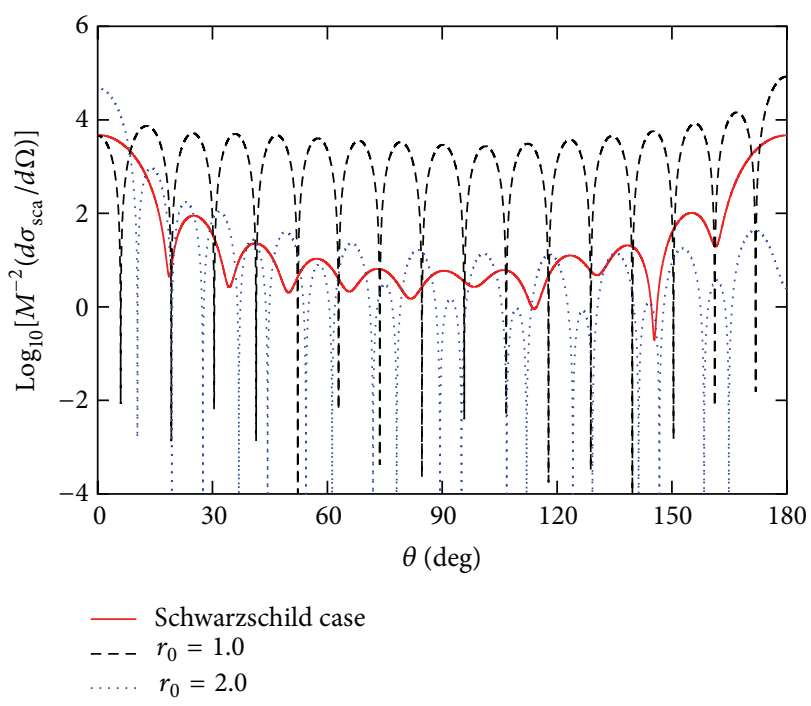

(c) Type 4

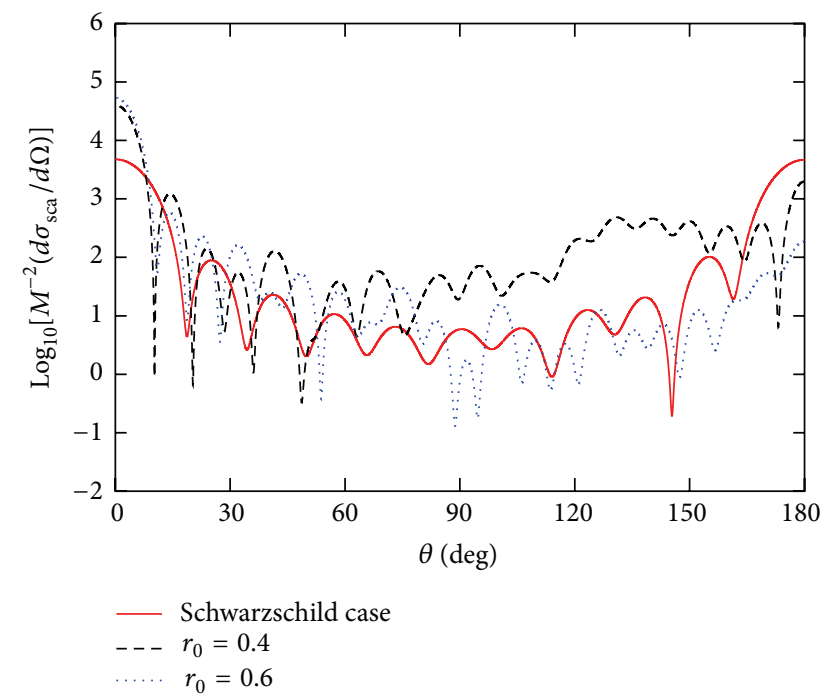

(b) Type 3

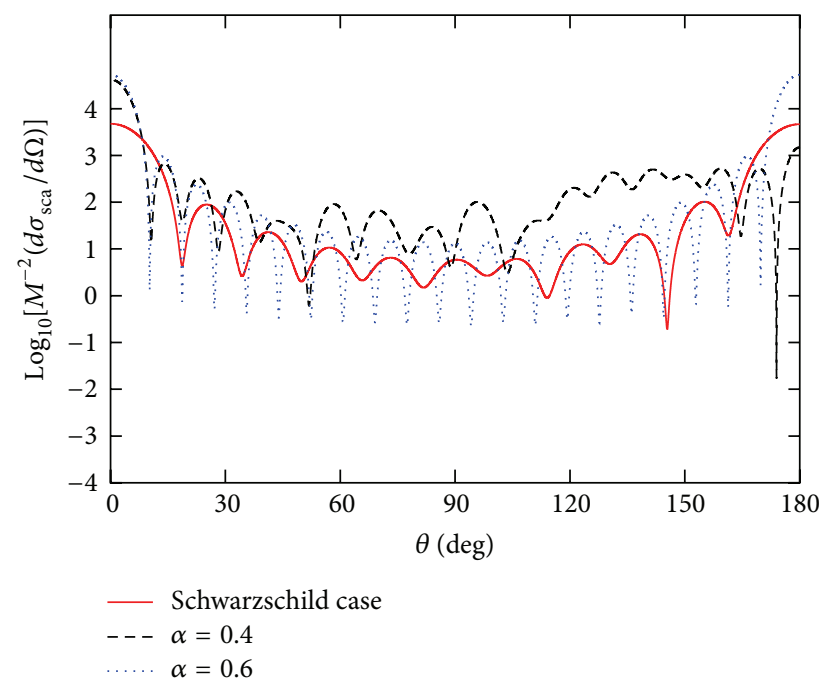

(d) Type 5

FIGURE 8: The behavior of the scattering cross section of types $2-5$ with a range of parameters $\alpha=0.1,0.4,0.6 ; r_{0}=0.1,0.2,0.3$; and $q=$ $0.1,0.4,0.6$. The red lines are Schwarzschild case.

weaker in the backward direction. In the angle of $90^{\circ}-180^{\circ}$, there is an irregular glory phenomenon; when $r_{0}=0.6$, the scattering flux is strengthened in both forward and backward directions.

The scattering cross section of type (6) has three parameters. In Figure 9 we show the scattering cross section for a range of parameters $b, c, \rho_{0}=0.2,0.4$. In order to see their contribution separately, we computed the scattering cross sections with two fixed parameters. We can see that (i) the effect of parameter $b$ is symmetry; both the forward and backward directions are strengthen. The width is narrow, and it means that we can see two obvious glory phenomena; (ii) the effect of parameter $c$ is also symmetry, and the scattering cross section is sensitive to parameter $c$; when $c=0.2$, the scattering is strengthened and the figure has several glory phenomena; (iii) when parameter $\rho_{0}=0.2$, the scattering in both forward and backward directions is strengthened and the width becomes narrow, and while $\rho_{0}=0.4$ the strength in backward direction is weaker.

\section{Conclusions}

In this paper, we have investigated the absorption and scattering cross section of the scalar wave from the regular black holes. In both low and high frequency region, we found that the parameter in different types of regular black holes makes the absorption cross section strengthened. The absorption cross sections oscillate around the geometric optical value in the high frequency region. We found that in most cases the scattering flux is strengthened and scattering cross section 


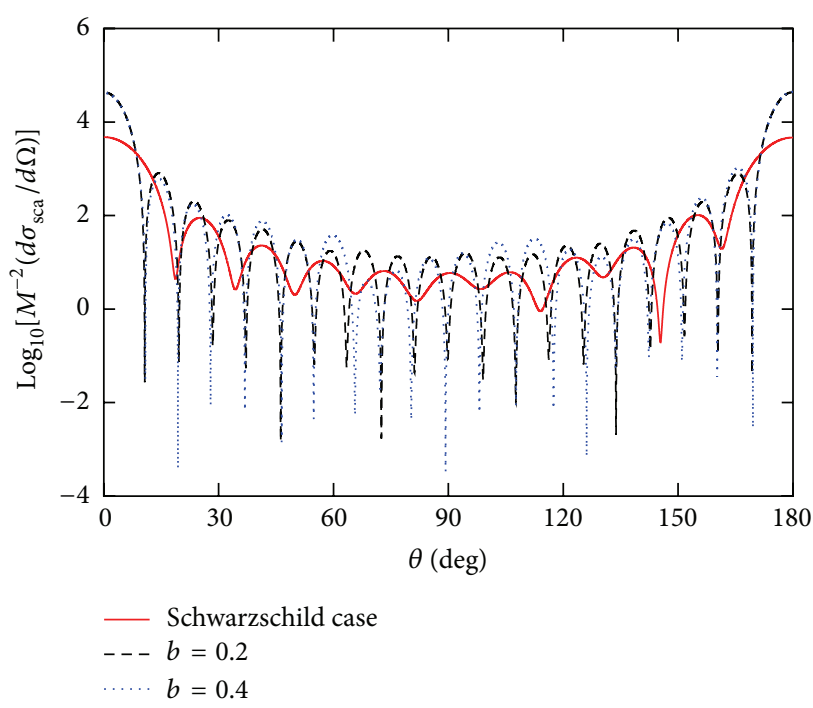

(a) $c=1 \rho_{0}=1$

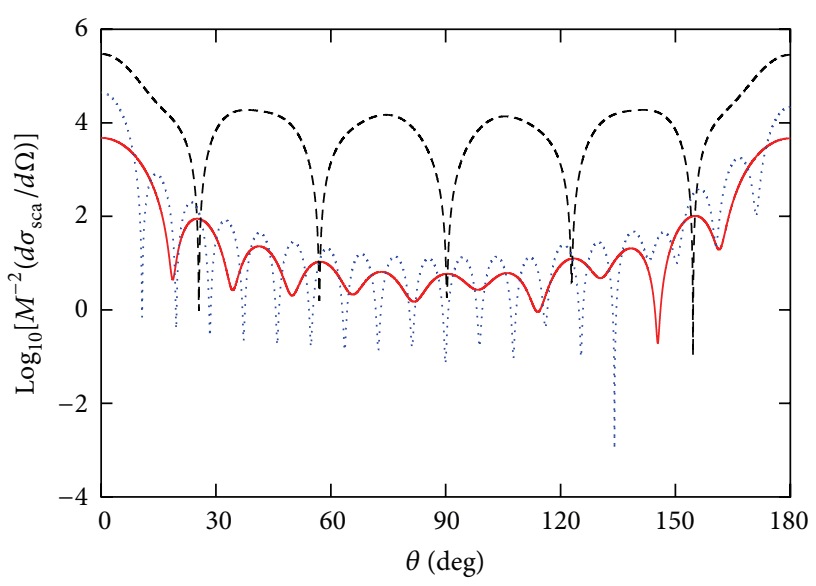

Schwarzschild case
$---c=0.2$
$\cdots \cdots \cdot c=0.4$

(b) $b=1 \rho_{0}=1$

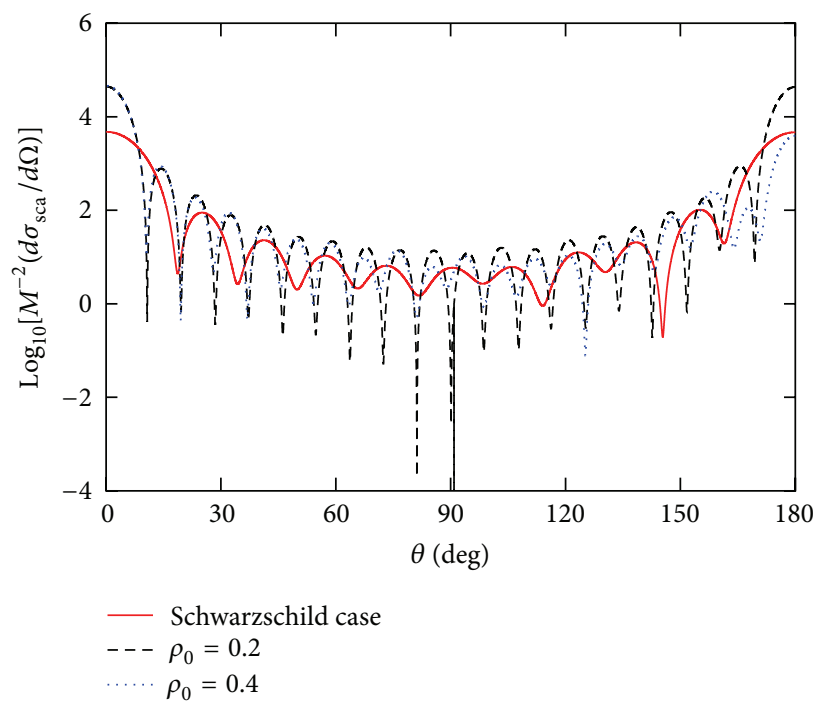

(c) $b=1 c=1$

FIGURE 9: The behavior of the scattering cross section of type 6 with a range of parameters $b=0.2,0.4 ; c=0.2,0.4 ; \rho_{0}=0.2,0.4$. The red lines are Schwarzschild case.

width becomes narrower in the forward direction. In the backward direction, the cross section is entirely different. In types 2, 4, and 5, scattering strength becomes partially strengthened and partially weaker. In types 3 and 5, there are glory phenomena in the region of $90^{\circ}-180^{\circ}$. We can also find average scattering strength in type 4 . But in type 6 , several glory phenomena are found in every direction. As a result, the glory phenomenon in type 6 is easier for astronomy observation.

\section{Conflict of Interests}

The authors declare that there is no conflict of interests regarding the publication of this paper.

\section{Acknowledgment}

This project is supported by the State Key Development Program for the Basic Research of China under Grant no. $2010 \mathrm{CB} 832803$.

\section{References}

[1] R. A. Matzner, "Scattering of massless scalar waves by a Schwarzschild 'singularity,' Journal of Mathematical Physics, vol. 9, no. 1, pp. 163-170, 1968.

[2] B. Mashhoon, "Scattering of electromagnetic radiation from a black hole," Physical Review D, vol. 7, no. 10, pp. 2807-2814, 1973.

[3] A. A. Starobinskiǐ, "Amplification of waves during reflection from a rotating 'black hole"' Soviet Physics JETP, vol. 37, no. 1, pp. 28-32, 1973. 
[4] A. A. Starobinskiǐ and S. M. Churilov, "Amplification of electromagnetic and gravitational waves scattered by a rotating 'black hole"' Soviet Physics JETP, vol. 38, p. 1, 1974.

[5] L. C. B. Crispino, A. Higuchi, and G. E. A. Matsas, "Quantization of the electromagnetic field outside static black holes and its application to low-energy phenomena," Physical Review D, vol. 63, no. 12, Article ID 124008, 14 pages, 2001.

[6] H. Liao, J. H. Chen, and Y. J. Wang, "Scattering of scalar wave from black hole in Hořava-Lifshitz gravity," International Journal of Modern Physics D, vol. 21, Article ID 125004, 2012.

[7] C. Doran, A. Lasenby, S. Dolan, and I. Hinder, "Fermion absorption cross section of a Schwarzschild black hole," Physical Review D, vol. 71, no. 12, Article ID 063502, 21 pages, 2005.

[8] J. Chen, H. Liao, and Y. Wang, "Absorption of massless scalar wave by high-dimensional Lovelock black hole," Physics Letters $B$, vol. 705, no. 1-2, pp. 124-128, 2011.

[9] V. P. Frolov and A. A. Shoom, "Scattering of circularly polarized light by a rotating black hole," Physical Review D, vol. 86, no. 2, Article ID 024010, 17 pages, 2012.

[10] J. H. Chen and Y. J. Wang, "Quasinormal modes of the scalar field in five-dimensional Lovelock black hole spacetime," Chinese Physics B, vol. 19, no. 6, Article ID 060401, 2010.

[11] L. Hao, J. H. Chen, and Y. J. Wang, "Absorption of a massless scalar wave from a Schwarzschild black hole surrounded by quintessence," Chinese Physics B, vol. 21, no. 8, Article ID 080402, 2012.

[12] T. Kobayashi and A. Tomimatsu, "Superradiant scattering of electromagnetic waves emitted from disk around Kerr black holes," Physical Review D, vol. 82, Article ID 084026, 11 pages, 2010.

[13] H. Okawa, K. Nakao, and M. Shibata, "Is super-Planckian physics visible? Scattering of black holes in 5 dimensions," Physical Review D, vol. 83, Article ID 121501, 5 pages, 2011.

[14] Y. Décanini, G. Esposito-Farèse, and A. Folacci, "Universality of high-energy absorption cross sections for black holes," Physical Review D, vol. 83, Article ID 044032, 10 pages, 2011.

[15] D. Batic, N. G. Kelkar, and M. Nowakowski, "Orbiting phenomena in black hole scattering," Physical Review D, vol. 86, Article ID 104060, 9 pages, 2012.

[16] J. M. Bardeen, "Non-singular general-relativistic gravitational collapse," in Proceedings of International Conference GR5, p. 174, USSR, Tbilisi, Georgia, 1968.

[17] E. Ayón-Beato and A. García, "The Bardeen model as a nonlinear magnetic monopole," Physics Letters B, vol. 493, no. 1-2, pp. 149-152, 2000.

[18] I. Dymnikova, "Regular electrically charged vacuum structures with de Sitter centre in nonlinear electrodynamics coupled to general relativity," Classical and Quantum Gravity, vol. 21, no. 18, pp. 4417-4428, 2004.

[19] E. Ayón-Beato and A. García, "Regular black hole in general relativity coupled to nonlinear electrodynamics," Physical Review Letters, vol. 80, no. 23, pp. 5056-5059, 1998.

[20] S. A. Hayward, "Formation and evaporation of nonsingular black holes," Physical Review Letters, vol. 96, no. 3, Article ID 031103, 2006.

[21] K. A. Bronnikov and J. C. Fabris, "Regular phantom black holes," Physical Review Letters, vol. 96, no. 25, Article ID 251101, 4 pages, 2006.

[22] K. A. Bronnikov, "Regular magnetic black holes and monopoles from nonlinear electrodynamics," Physical Review D, vol. 63, no. 4, Article ID 044005, 6 pages, 2001.
[23] W. Berej, J. Matyjasek, D. Tryniecki, and M. Woronowicz, "Regular black holes in quadratic gravity," General Relativity and Gravitation, vol. 38, no. 5, pp. 885-906, 2006.

[24] A. Borde, "Regular black holes and topology change," Physical Review D, vol. 55, no. 12, pp. 7615-7617, 1997.

[25] V. Ferrari and B. Mashhoon, "Oscillations of a black hole," Physical Review Letters, vol. 52, no. 16, pp. 1361-1364, 1984.

[26] S. Okuzumi and M.-A. Sakagami, "Quasinormal ringing of acoustic black holes in Laval nozzles: numerical simulations," Physical Review D, vol. 76, no. 8, Article ID 084027, 2007.

[27] Y. Décanini, G. Esposito-Farèse, and A. Folacci, "Universality of high-energy absorption cross sections for black holes," Physical Review D, vol. 83, no. 4, Article ID 044032, 2011.

[28] K. Gottfried and T.-M. Yan, Quantum Mechanics: Fundamentals, Springer, New York, NY, USA, 2nd edition, 2004.

[29] S. R. Dolan, E. S. Oliveira, and L. C. B. Crispino, "Scattering of sound waves by a canonical acoustic hole," Physical Review D, vol. 79, no. 6, Article ID 064014, 9 pages, 2009.

[30] R. A. Matzner, C. Dewitte-Morette, B. Nelson, and T.-R. Zhang, "Glory scattering by black holes," Physical Review D, vol. 31, no. 8, pp. 1869-1878, 1985.

[31] L. C. B. Crispino, S. R. Dolan, and E. S. Oliveira, "Scattering of massless scalar waves by Reissner-Nordström black holes," Physical Review D, vol. 79, no. 6, Article ID 064022, 9 pages, 2009. 

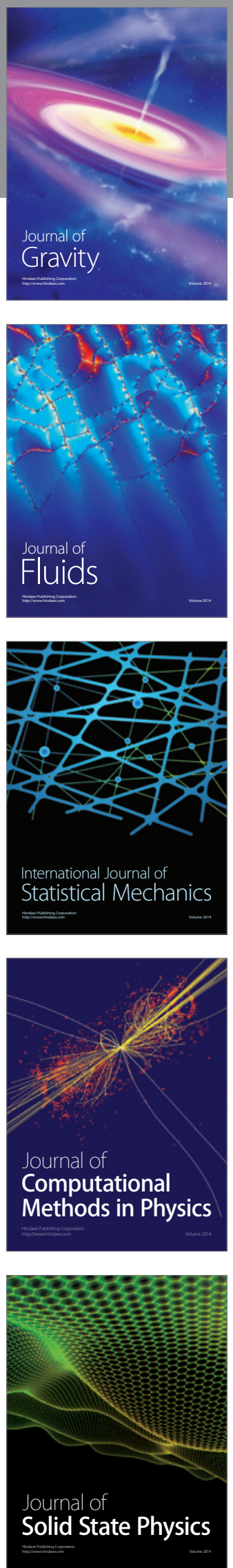

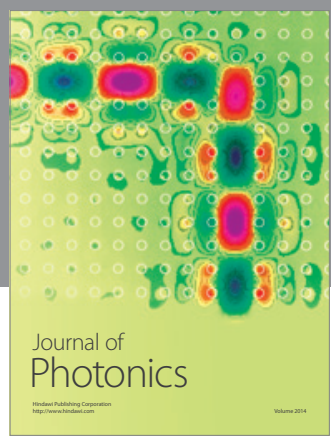

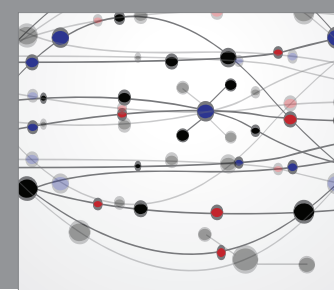

The Scientific World Journal

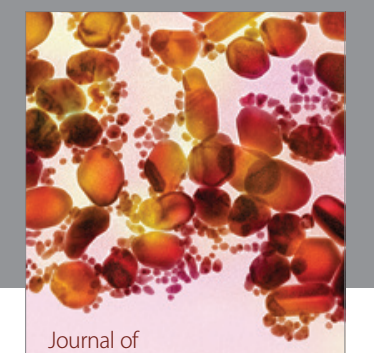

Soft Matter
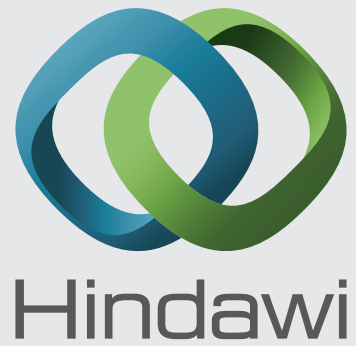

Submit your manuscripts at

http://www.hindawi.com
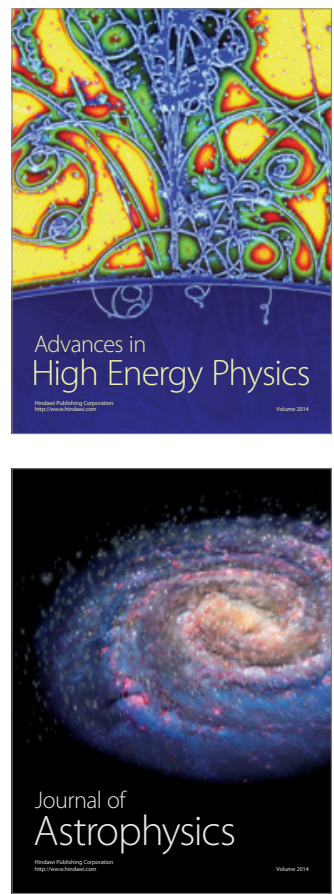
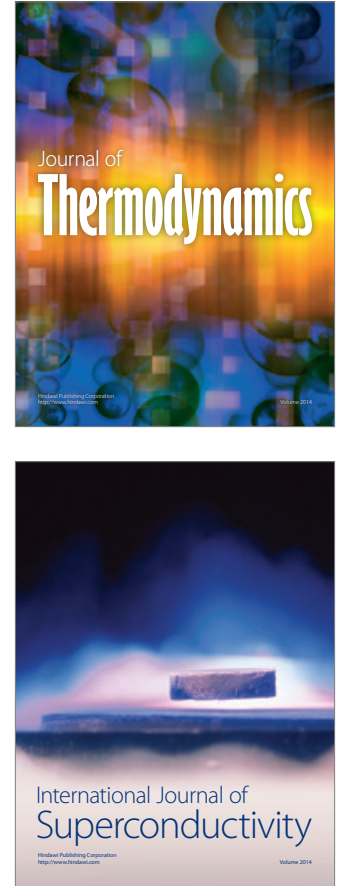
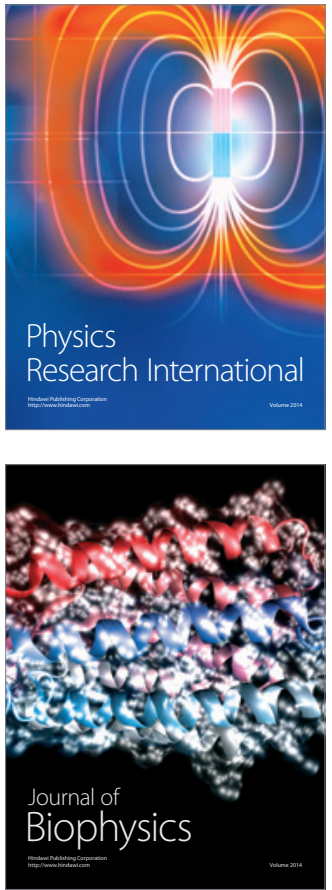
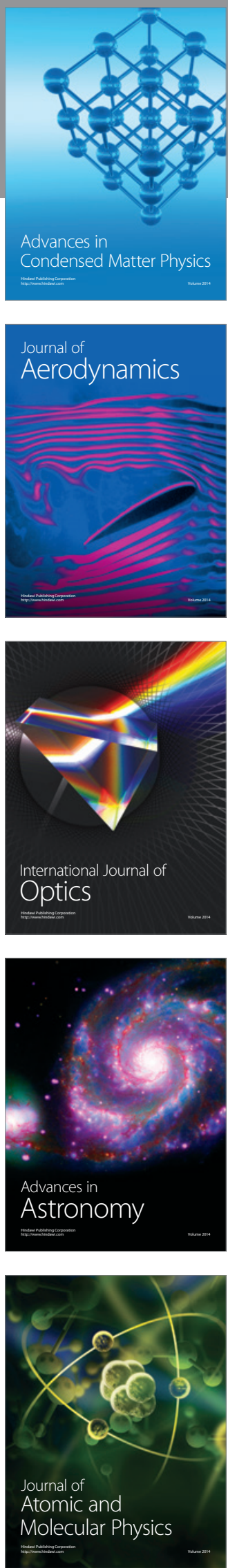